\title{
Sinusoidal Movement of a Grating Across the Monkey's Fingerpad: Representation of Grating and Movement Features in Afferent Fiber Responses ${ }^{1}$
}

\author{
A. W. Goodwin and J. W. Morley \\ Department of Anatomy, University of Melbourne, Parkville, Victoria 3052, Australia
}

Gratings of alternating grooves and ridges were moved sinusoidally back and forth across the monkey's fingerpad. Each grating was completey specified by its spatial period and the movement by its peak speed: together these determined the peak temporal frequency at which grating ridges passed over the skin. Responses of cutaneous, mechanoreceptive afferents innervating the fingerpad were characterized in terms of these 3 parameters. Slowly adapting afferents (SAs), rapidly adapting afferents (RAs), and Pacinian afferents (PCs) had different characteristics. The responses (mean cyclic discharge rates) of the SAs increased when the spatial period of the grating increased (and peak speed of movement remained constant) but did not change with changes in the peak speed of the movement (while the spatial period of the grating remained constant). Conversely, the responses of the PCs increased when the peak speed of movement increased (and the spatial period remained constant) but were relatively insensitive to changes in the spatial period of the grating (while the peak speed remained constant). The responses of the RAs increased as the spatial period of the grating increased (and peak speed remained constant) and also increased as the peak speed of movement increased (and the grating spatial period remained constant). When the peak temporal frequency of the grating ridges was held constant, the responses of all 3 afferent groups changed with changes in the grating spatial period or in the peak speed of movement. Information about the spatial features of the grating, independent of the peak speed of movement, was present in the SA population response and in the ratios of the RA and PC population responses. Information about the peak speed of movement, independent of the spatial period of the grating, was present in the PC population response and could be extracted from the RA population response.

To discriminate 2 differently textured surfaces, such as woollen fabric and cotton fabric, human observers typically rub their fingertips back and forth across the surfaces. The relative move-

Received July 9, 1986; revised Dec. 9, 1986; accepted Jan. 8, 1987

This work was supported by a grant from the National Health and Medical Research Council of Australia. We thank Neil Ratcliffe, Colin Clarke, and Alf Medoro for technical assistance, Kati Bromley for the illustrations, and Janet Bennett for typing the manuscript.

Correspondence should be addressed to Dr. A. W. Goodwin, Department of Anatomy, University of Melbourne, Grattan Street, Parkville, Victoria 3052, Australia.

Copyright @ 1987 Society for Neuroscience $0270-6474 / 87 / 072168-13 \$ 02.00 / 0$ ment of the fingers and the surface activates the mechanoreceptor populations in the skin. Information within these population discharges allows the observer to make judgments about the texture. A convenient textured surface for experimental study is a grating of alternating grooves and ridges. Lederman and Taylor (1972) have shown that a sequence of such gratings can be scaled for roughness, and Morley et al. (1983) have shown that the human can discriminate, at the $75 \%$ level, 2 gratings that differ in spatial period by about $5 \%$. The pattern of finger movement used by the subjects in the discrimination experiments was approximately sinusoidal, but different subjects used different speeds. Common experience suggests that we can assess the texture of a surface independently of the characteristics of the exploratory movements. This is supported by Lamb's (1983a) observation that a human's capacity to discriminate dot patterns that differ in size is independent of the movement profile. There is also indirect evidence suggesting that the discriminative capacities would be the same if the fingers were held still and the grating were scanned across them sinusoidally (Day and Dickenson 1979; Lederman, 1981; Lamb, 1983a). Apparently the only requirement is movement between the grating and the skin, and the discharge of the cutaneous, mechanoreceptor populations contains sufficient information for the subject to extract details about the spatial characteristics of the surface that are independent of the movement characteristics.

The responses of cortical neurons in the postcentral gyrus of the monkey, when the fingertips are scanned across a grating, have been described by Darian-Smith et al. (1985). At the peripheral nerve level of the monkey, the responses of cutaneous mechanoreceptive afferents have been described for dot patterns moving at constant specd (Darian-Smith ct al., 1980; Johnson and Lamb, 1981; Lamb, 1983b) and for stationary gratings (Phillips and Johnson, 1981). However, the only data available on the responses of the primary afferents to moving gratings are those of Darian-Smith and Oke (1980), who used gratings moving over the skin at constant speed. To our knowledge there has been no study of the responses of cutaneous afferents to gratings moved back and forth across the receptive field, which is a closer approximation to the finger movements used by human observers. In the present experiments, gratings were moved sinusoidally back and forth across the receptive fields of cutaneous mechanoreceptive afferents in the fingerpads of anesthetized monkeys. The spatial period of the grating and the speed of movement were varied independently. Responses of individual primary afferents were recorded and characterized and the responses of the afferent populations synthesized. 


\section{Materials and Methods}

Single-fiber recording. Six macaques, $4 \mathrm{M}$. nemestrina and $2 \mathrm{M}$. mulatta, weighing between 3 and $5 \mathrm{~kg}$ were used. The techniques for recording from single fibers were similar to those used previously (Goodwin et al., 1981) and are described ony briefly here.

The monkeys were tranquilized with ketamine hydrochloride, and anesthesia was induced by intravenous sodium pentobarbital and maintained during the experiments by intraperitoneal sodium pentobarbital. Body tcmpcrature was maintained at about $37^{\circ} \mathrm{C}$ by a heating blanket, and antibiotic cover was provided by amoxycillin sodium $(12 \mathrm{mg} / \mathrm{kg}$ every $6 \mathrm{hr}$ ). Eight recording sites were used per animal, namely, the median and ulnar nerve in both upper arms and in both lower arms. For each nerve the upper recording site was used first, and at least one week elapsed before use of the lower site to ensure that no recordings were made from degenerating fibers. At the end of the experiment the dissection was thoroughly cleaned and sutured, and the animal given antibiotic ( $225 \mathrm{mg}$ benzathine penicillin, $150 \mathrm{mg}$ procaine penicillin, and $90 \mathrm{mg}$ potassium penicillin). At least 6 weeks elapsed between experiments on the 2 nerves in the same arm to allow complete healing of the first wound. In each dissection only a small proportion of the nerve was used, so that the animals suffered no obvious sensory loss or paralysis.

Receptive field characterization. Oncc a single fibcr had been isolated, the location of the receptive field center was determined by a series of graded von Frey hairs. In this study we used only cutaneous afferents that had receptive fields on the central portion of the fingerpad. To classify the afferent, the receptive field was stimulated with a vibratory probe (Chubbuck, 1966). Slowly adapting fibers (SAs) responded to static indentation of the skin. The SAs in our sample formed a single, homogeneous group and could not be divided into SAI and SAII afferents. The properties of the SAs were consistent with those reported for the SAI group in humans (Johansson, 1978) and with those reported by others for SAs in the monkey (Phillips and Johnson, 1981). Rapidly adapting fibers (RAs) and Pacinian afferents (PCs) were distinguished on the basis of their vibratory tuning curves (Talbot et al., 1968). The "receptive fields" of the PCs extended beyond the central region of the fingerpad, but in all cascs they were most sensitive to stimuli on one finger (rather than other parts of the hand) and usually were most sensitive to stimuli on the 2 most distal phalanges of that finger. Data were collected from 22 SAs, 30 RAs, and 7 PCs.

Stimulation with gratings moved sinusoidally. The stimulus in these experiments was a grating of alternating grooves and ridges moved back and forth sinusoidally over the receptive field of the afferent. Nyloprint gratings (nylon backed on steel) were prepared commercially by an ultraviolet photopolymerization method and were made as close to a "rectangular pulse" grating as possible (see Fig. 1 of Morley et al., 1983, for details of the profile). The ratio of the groove width to ridge width was held constant at a nominal value of 7 , and the spatial period had nominal values of $0.75,1,1.25,1.5,1.75,2$, or $3 \mathrm{~mm}$. All gratings were examined microscopically to ensure that there were no imperfections, and the actual values of the periods and groove width to ridge width ratios were measured: These were found to be respectively $0.77 \mathrm{~mm}$ and $7.9,1.04 \mathrm{~mm}$ and $6.5,1.28 \mathrm{~mm}$ and $6.2,1.57 \mathrm{~mm}$ and $7.3,1.80$ $\mathrm{mm}$ and $6.1,2.04 \mathrm{~mm}$ and 6.3 , and $3.14 \mathrm{~mm}$ and 6.8 . In all cases the groove was sufficiently deep that the monkey's finger did not touch the bottom of the groove.

The steel-backed gratings were magnetically attached to a stimulator that moved back and forth sinusoidally with a peak-to-peak amplitude of $80 \mathrm{~mm}$. Sinusoidal motion was achieved by a mechanical yoke ("scotch yoke") driven by a constant-speed DC motor (Goodwin et al., 1985). The period of the sinusoid could be varied from 0.5 to $18 \mathrm{sec}$

As shown in Figure 1, the monkey's hand was taped to a Perspex plate and the finger to be stimulated was pressed into a bed of Plasticene (modeling clay) with the fingernail secured by cotton thread so that there was no movement between the plate and the dorsal aspect of the finger. However, care was taken that the fingerpad was free from Plasticene, both ventrally and laterally, and able to move normally when stimulated. The Perspex plate was attached to a micrometer so that it could be raised and lowered precisely, with a dial indicator measuring the movement. Initially, the grating was positioned about $1 \mathrm{~mm}$ above the receptive field. Prior to stimulation the micrometer was used to raise the plate until the fingerpad just touched the grating as determined visually. At the midpoint of the sinusoidal motion, the center of the receptive field was located at the center of the grating, which was 100 $\mathrm{mm}$ long and $20 \mathrm{~mm}$ wide. During stimulation the finger was indented

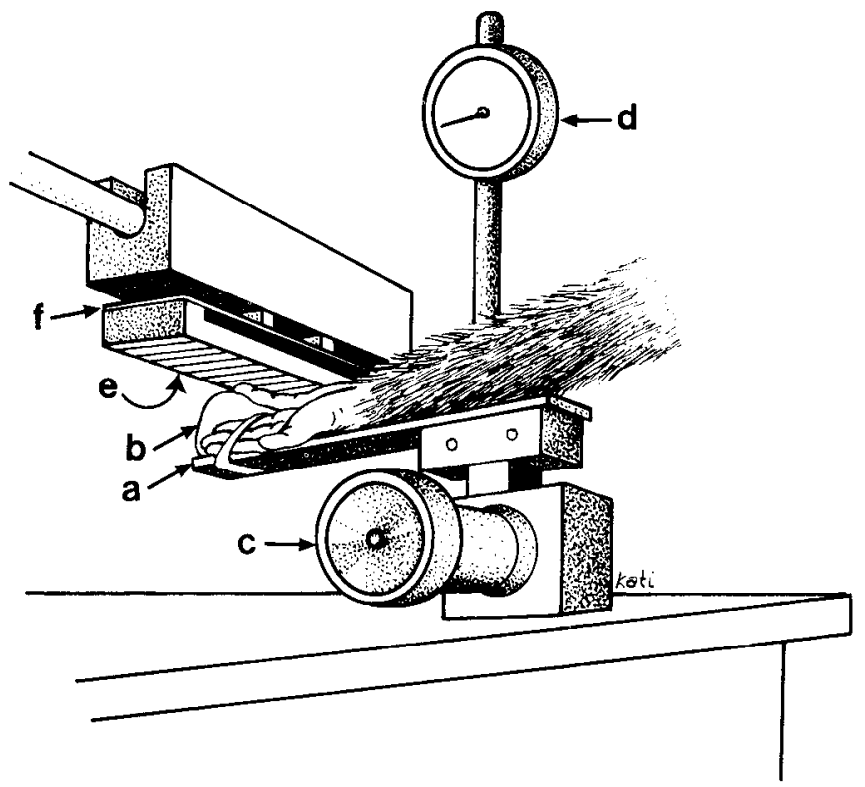

Figure 1. Method of stimulating the afferent. The monkcy's hand was taped to a Perspex plate $(a)$ attached to a micrometer $(c)$ that could be raised and lowered by an amount read on the dial indicator $(d)$. The finger to be stimulated was secured in a bed of Plasticene (modeling clay) $(b)$. The grating (e) moved sinusoidally over the fingerpad, tangentially to the skin at the center of the receptive field. Contact force was measured by the strain gauge $(f)$.

$1 \mathrm{~mm}$ into the grating, from the point of contact, as measured by the dial indicator (resolution, $0.01 \mathrm{~mm}$ ). Care was taken to ensure that the plane of the grating was tangential to the fingerpad at the receptive field center and that the grating was not close to skin creases or interphalangeal creases. To monitor the contact force between the finger and the grating, the grating was mounted on the stimulator via a strain gauge bridge with a resolution of $0.0025 \mathrm{~N}$. Contact between the finger and the grating could also be ascertained by detecting a contact force of approximately $0.003 \mathrm{~N}$, but this was found to be no more precise than visual assessment and less convenient. To minimize transfer of vibration, the monkey and the stimulator were located on separate tables linked only by a rubber coupling. Vibration in the stimulator itself was minimized by rubber mounts and belt couplings (Goodwin et al., 1985).

For a particular combination of stimulus parameters, the experimental sequence was as follows. With the grating positioned about $1 \mathrm{~mm}$ above the receptive field, the sinusoidal motion of the grating was initiated. The hand support was raised until the finger just touched the grating and then raised an additional $1 \mathrm{~mm}$. A run of data collection then commenced and continued for about 20 cycles of sinusoidal motion. After the run, the hand support was lowered until the finger was well clear of the grating. At least 1 min elapsed before the next run of data collection commenced. For each grating spatial period, a number of preselected sinusoidal temporal periods were used; Table 1 gives the full range of combinations. The range of parameters was chosen to cover the range of speeds used by human subjects and the range of frequencies used in previous vibratory studies of mechanoreceptors. To collect data from an afferent with the full range of parameter combinations (including variables in the companion papers) would be impractically timeconsuming. Thus, to optimize the yield from each afferent the parameters were not varied in the same order each time, nor were they varied in a truly random order: Rather, for each afferent the order was chosen to provide the most useful data from that afferent first and so on. To guard against possible time effects such as adaptation and possible interactions between successive runs with different parameters (even though at least 1 min elapsed between runs), a number of test runs were included for each afferent. These test runs consisted of identical conditions presented at successive times during the experiment, to ensure that the afferent was not exhibiting any long-term adaptation, and also of identical conditions presented after a range of conditions, to ensure that the test response was not affected by the parameters of the preceding run.

An infrared detector system on the stimulator provided one TTL 


\begin{tabular}{|c|c|c|c|c|c|c|c|}
\hline \multirow{2}{*}{$\begin{array}{l}\text { Fre- } \\
\text { quency }\end{array}$} & \multicolumn{7}{|c|}{ Spatial period (mm) } \\
\hline & 0.75 & 1.0 & 1.25 & 1.5 & 1.75 & 2.0 & 3.0 \\
\hline \multicolumn{8}{|l|}{$10 \mathrm{~Hz}$} \\
\hline$s$ & & & & & & 20 & 30 \\
\hline$T$ & & & & & & 12.566 & 8.378 \\
\hline \multicolumn{8}{|l|}{$20 \mathrm{~Hz}$} \\
\hline$s$ & 15 & 20 & 25 & 30 & 35 & 40 & 60 \\
\hline$T$ & 16.755 & 12.566 & 10.053 & 8.378 & 7.181 & 6.283 & 4.189 \\
\hline \multicolumn{8}{|l|}{$40 \mathrm{~Hz}$} \\
\hline$s$ & 30 & 40 & 50 & 60 & 70 & 80 & 120 \\
\hline$T$ & 8.378 & 6.283 & 5.027 & 4.189 & 3.590 & 3.142 & 2.094 \\
\hline \multicolumn{8}{|l|}{$80 \mathrm{~Hz}$} \\
\hline$s$ & 60 & 80 & 100 & 120 & 140 & 160 & 240 \\
\hline$T$ & 4.189 & 3.142 & 2.513 & 2.094 & 1.795 & 1.571 & 1.047 \\
\hline \multicolumn{8}{|l|}{$160 \mathrm{~Hz}$} \\
\hline$s$ & 120 & 160 & 200 & 240 & 280 & 320 & 480 \\
\hline$T$ & 2.094 & 1.571 & 1.257 & 1.047 & 0.898 & 0.785 & 0.524 \\
\hline \multicolumn{8}{|l|}{$320 \mathrm{~Hz}$} \\
\hline$s$ & 240 & 320 & 400 & 480 & & & \\
\hline$T$ & 1.047 & 0.785 & 0.628 & 0.524 & & & \\
\hline
\end{tabular}

Each column represents one of the 8 grating spatial periods $(p$, in $\mathrm{mm}$ ) used. For each grating a range of peak speeds $(s$ in $\mathrm{mm} / \mathrm{sec}$ ) was chosen so that the resulting peak temporal frequencies of the grating ridges $(f$, in Hz) were $10,20,40$, 80,160 , and 320. The parameters are related by $s-p f$. Each peak speed $s$ has an equivalent cycle period $(T$, in sec), given by $T=251.3 / \mathrm{s}$

logic pulse for every $6^{\circ}$ of the cycle and 1 pulse for each complete cycle. The time of occurrence of these digital events and the time of occurrence of action potentials in the afferent were collected by a laboratory computer with a resolution of $0.1 \mathrm{msec}$. In addition, the computer collected 2 analog events via an analog-to-digital converter sampling each channel at $50 \mathrm{~Hz}$. These were the output of the strain gauge bridge measuring the contact force between the finger and the grating, and the output of an optical transducer measuring the position of the grating.

\section{Results}

As the grating moved cyclically back and forth across the afferent's receptive field, the afferent responded cyclically in synchrony with the movement. This is illustrated for an SA in Figure $2 A$ for 3 cycles of movement. In each cycle the grating started at one extreme position $(-40 \mathrm{~mm})$ at zero speed, accelerated to reach a maximum speed (in this case $60 \mathrm{~mm} / \mathrm{sec}$ ) at the center position $(0 \mathrm{~mm})$, and then decelerated to reach the opposite extreme position ( $+40 \mathrm{~mm}$ ), again at zero speed. The direction of movement then reversed, and the grating accelerated, again reaching maximum speed at the center position and then decelerating to reach the extreme position $(-40 \mathrm{~mm})$ at zero speed. As the stimulus is cyclic, the characteristics of the discharge are most easily seen in a cycle histogram, as shown for the same SA in Figure $2 B$. For all the afferents we studied the discharge was cyclic in synchrony with the stimulus for all values of the stimulus parameters. For the SA illustrated in Figure 2, the response profile closely reflected the speed profile of the grating. However, this was not always the case, and the shape of the profile was a complex function of the particular afferent and the stimulus parameters. This issue is dealt with in the following paper (Morley and Goodwin, 1987).

When a textured surface is rubbed over a human fingertip, 2 parameters that are important in the assessment of that surface are its spatial characteristics and the characteristics of the move- ment. In this paper we describe the effects on the primary afferent discharge of the corresponding 2 parameters. Since the gratings have a groove width to ridge width ratio that is held constant at the nominal value of 7 , the surface is completely specified by a single parameter, namely, the spatial period of the grating. The movement is always sinusoidal with a peak-topeak amplitude of $80 \mathrm{~mm}$, so that the movement profile is also completely specified by a single parameter, either the temporal period of the sinusoid or, equivalently, the peak speed: the peak speed was used here because, as will be seen, the analyis is simplified. The peak speed (in $\mathrm{mm} / \mathrm{sec}$ ) is 251.3 divided by the temporal period in seconds. Table 1 shows the equivalent temporal periods for the various peak speeds used.

The cycle histograms in Figure 3 illustrate the effect on an RA's response to changes in the stimulus parameters. For the top row of histograms the surface spatial period was held constant at $3 \mathrm{~mm}$ while the peak speed was doubled from 120 to $240 \mathrm{~mm} / \mathrm{sec}$. This increase in peak spccd resultcd in an incrcase in the peak discharge rate of the fiber. The mean cyclic discharge rate (i.e., the average number of impulses elicited in 1 cycle divided by the cycle period) also increased, from 162 to 223 impulses/sec. For the left column of histograms the peak speed remained constant at $120 \mathrm{~mm} / \mathrm{sec}$ while the spatial period of the surface was halved from 3 to $1.5 \mathrm{~mm}$. In this case, the peak discharge rate of the fiber decreased. The mean cyclic discharge rate also decreased, from 162 to 112 impulses/sec. Thus, the discharge rate of this fiber depended both on the spatial period of the surface and on the peak speed of movement, under these particular conditions decreasing with a decrease in spatial period or a decrease in peak speed.

Were these changes in response statistically significant? As can be seen in the 4 cycles of Figure $2 B$, the patterns of impulses were highly repeatable from cycle to cycle, and this was typical 


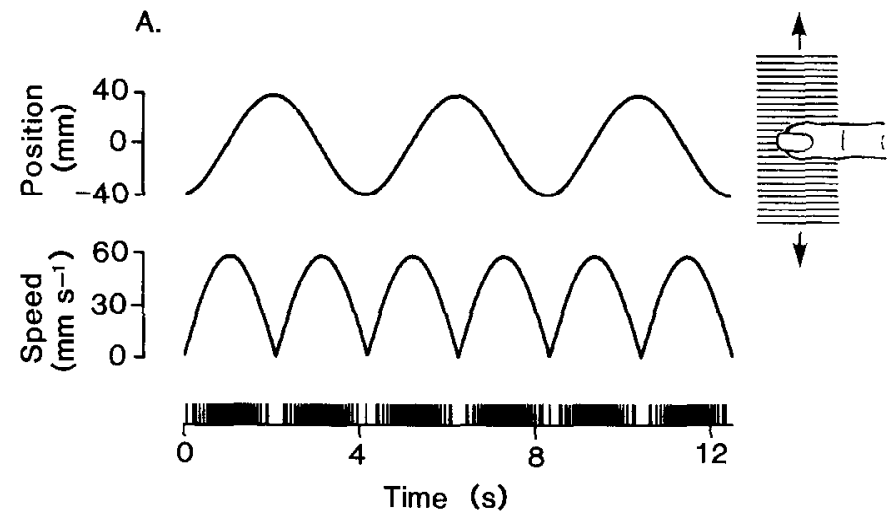

B.

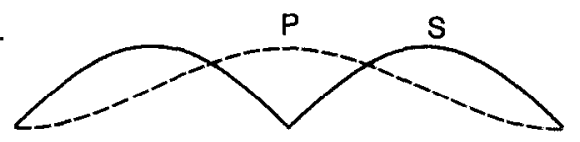

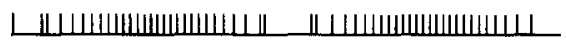

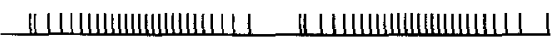

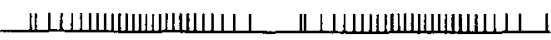

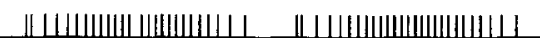

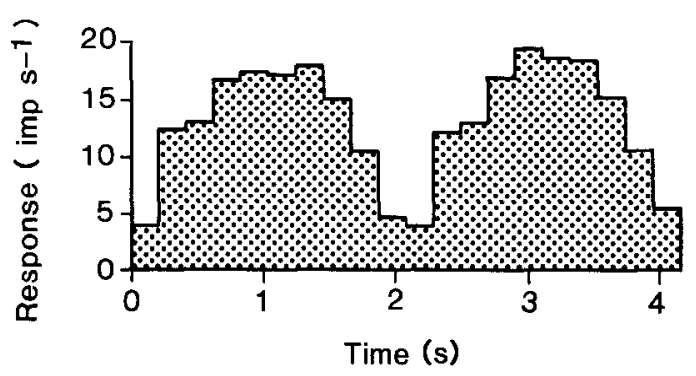

Figure 2. Response of an SA to a grating with a spatial period of 3 $\mathrm{mm}$ moving sinusoidally over the receptive field. The sinusoid had a peak-to-peak amplitude of $80 \mathrm{~mm}$ and a temporal period of $4.189 \mathrm{sec}$. $A$, Three successive cycles of the stimulus. Top and middle traces show the instantaneous position and speed of the grating, respectively, and vertical strokes in the bottom trace indicate the time of occurrence of action potentials. $B$. Cycle histogram. Broken and solid lines in top trace show the instantaneous position and speed, respectively, during each cycle. Vertical strokes in middle show the time of occurrence of action potentials during the first 4 cycles. Poststimulus time histogram (PSTH) at bottom shows the cyclic response averaged over 15 cycles (bin width $0.209 \mathrm{sec}$ ).

of all the afferents we studied. For the SA in Figure $2 B$, the mean cyclic discharge rate was $13.1 \pm 0.18$ impulses/sec (mean \pm SEM, $n=15$ ). In Figure 3 , the mean cyclic discharge rates and their SEs were $162 \pm 1.43(n=10), 223 \pm 2.45(n=18)$, and $112 \pm 1.49(n=15)$. These responses are clearly significantly different ( $t$ test, $p \ll 0.01$ ). The above SEs of about $1 \%$ of the mean cyclic discharge rate are typical of our afferents, so that further unnecessary statistics are not included in Figures 4, 6, 8 , etc.

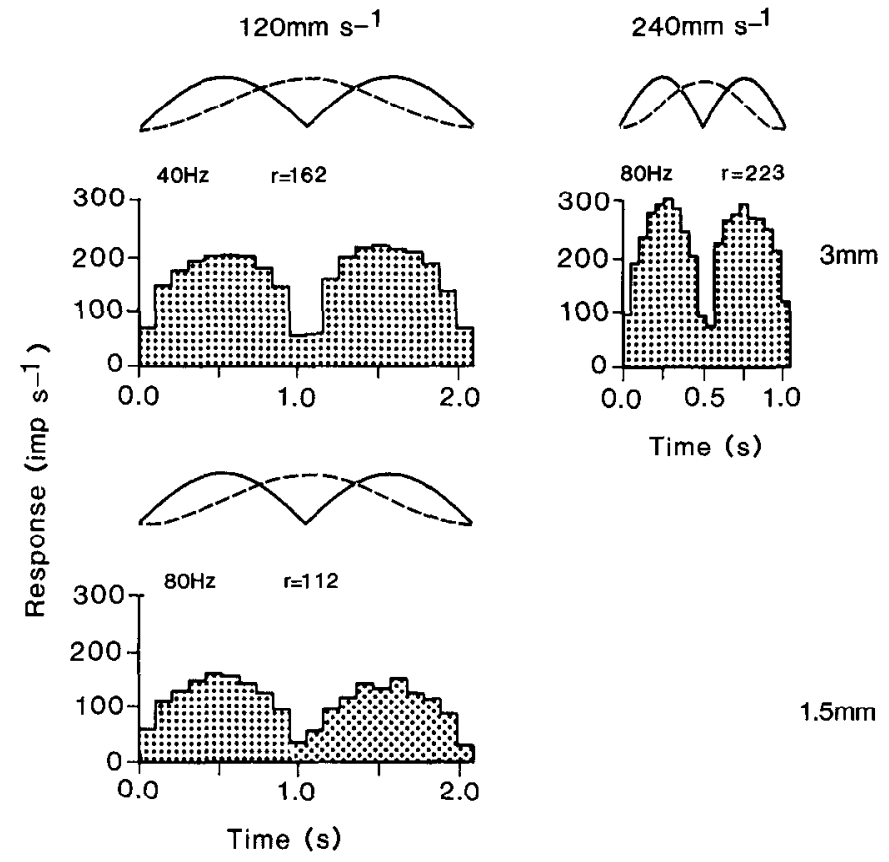

Figure 3. Cycle histograms showing the effect of changes in stimulus parameters on the response of an RA. The spatial period of the grating was $3 \mathrm{~mm}$ for the top row and $1.5 \mathrm{~mm}$ for the bottom. The peak speed of movement was $120 \mathrm{~mm} / \mathrm{sec}$ for the left column and $240 \mathrm{~mm} / \mathrm{sec}$ for the right. Values above each histogram show the peak temporal frequency of the grating ridges (in $\mathrm{Hz}$ ) and the mean cyclic responses, $r$, impulses per second (average number of impulses elicited in a cycle divided by the cycle period). A change in any of the stimulus parameters produced a change in the RA's response. Broken and solid lines above each histogram show instantaneous position and speed, respectively. Number of cycles averaged and bin widths are, respectively, 10 and $0.105 \mathrm{sec}$ (top left), 18 and $0.052 \mathrm{sec}$ (top right), and 15 and $0.105 \mathrm{sec}$ (bottom left).

The situation is further complicated by considering an alternative description of the temporal characteristics of the stimulus. The rate at which successive ridges of the grating pass over the center of the fiber's receptive field depends on both the spatial period and the speed of movement of the grating. DarianSmith and Oke (1980) found that the responses of cutaneous afferents from the fingertip to gratings moving over their receptive fields at constant speed (linearly) were most conveniently described in terms of this parameter of the stimulus. For linear motion, the temporal frequency of the grating ridges is given by the movement speed divided by the grating spatial period. When the movement of the grating is sinusoidal, the temporal frequency of the ridges is approximately sinusoidal (see Appendix of following paper) and for our stimulus is best described by its peak value: peak temporal frequency $(\mathrm{Hz})=$ peak speed $(\mathrm{mm} /$ sec)/spatial period ( $\mathrm{mm}$ ). A change in either the peak speed or the spatial period of the grating will result in a change in the peak temporal frequency of the ridges. Thus, in the top row of Figure 3, doubling the peak speed doubled the peak temporal frequency of the ridges from 40 to $80 \mathrm{~Hz}$; in the left column, halving the spatial period also doubled the peak temporal frequency of the ridges from 40 to $80 \mathrm{~Hz}$. On the other hand, comparing the top-right and bottom-left histograms shows that both the peak speed of movement and the spatial period of the grating could be changed in such a way that the peak temporal frequency of the ridges remained constant. Obviously, any 2 of 


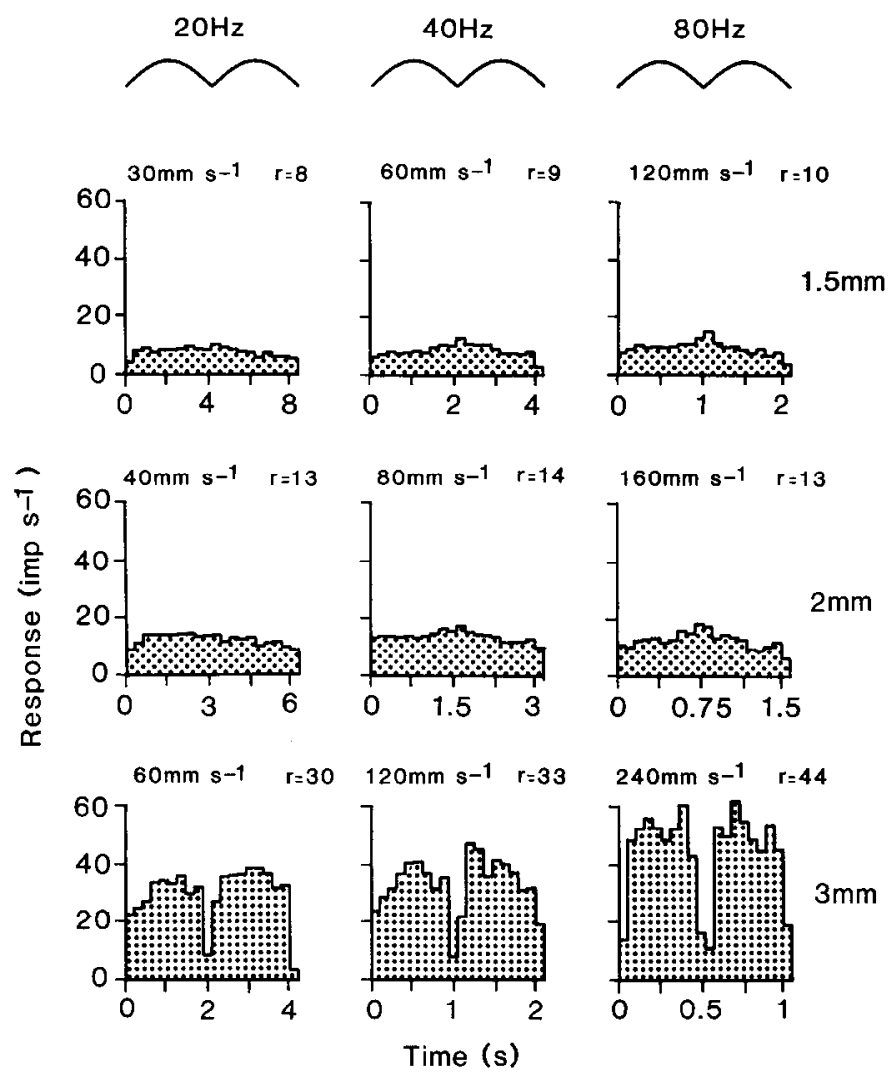

Figure 4. Matrix of cycle histograms for a typical SA. In each row the spatial period of the grating was held constant. In each column the peak temporal frequency of the grating ridges was held constant. Thus, within each row the peak speed of movement increasd from left to right with the values shown above each histogram. The mean cyclic response (in impulses/sec) is shown above each histogram as $r$. Note that the time scales are different for the different histograms. Moving from left to right in the 1st, 2nd, and 3rd rows successively, the number of cycles averaged and bin widths for the 9 histograms are, respectively, 9 and $0.419 \mathrm{sec}, 10$ and $0.209 \mathrm{sec}, 17$ and $0.105 \mathrm{sec}, 9$ and $0.314 \mathrm{sec}, 14$ and $0.157 \mathrm{sec}, 16$ and $0.079 \mathrm{sec}, 13$ and $0.209 \mathrm{sec}, 16$ and $0.105 \mathrm{sec}$, and 17 and $0.052 \mathrm{sec}$.

the parameters (spatial period, peak speed, and peak temporal frequency) can be considered as independent, and the third dependent parameter is defined by the relationship given above. This complicates analysis because we cannot assume a priori which, if any, of the parameters are independently processed by the nervous system, and changes in any one of the parameters will produce concomitant changes in one of the other parameters. An effective method of analysis in such cases is parametric, in which one of the parameters is held constant while the response of the afferent is characterized as a function of each of the other variables. As an illustration, consider the RA in Figure 3. If the response of this unit depended only on the peak speed of movement, then the top left and bottom responses would be the same. This is obviously not the case. If the response depended only on the spatial period of the grating, then the top left and right responses would be the same. This is not the case either. Finally, if the responses depended only on the peak temporal frequency, then the top right and bottom left responses would be the same. This too is not the case. Thus, for this unit, the response cannot be simply described in terms of one of the variables. A detailed parametric analysis of the units studied by us follows.
Table 2. Average response (impulses/sec) of the SA population for the various combinations of stimulus parameters

\begin{tabular}{llrrrrrr} 
Fre- & \multicolumn{6}{l}{ Spatial period (mm) } \\
\cline { 2 - 8 } quency & 0.75 & 1.0 & 1.25 & 1.5 & 1.75 & 2.0 & 3.0 \\
\hline $10 \mathrm{~Hz}$ & & & & & & & \\
$\mu$ & & & & & & 20 & 40 \\
$\sigma$ & & & & & & 13 & 16 \\
$n$ & & & & & & 8 & 18 \\
$20 \mathrm{~Hz}$ & & & & & & & \\
$\mu$ & 5 & 7 & 12 & 16 & 25 & 26 & 43 \\
$\sigma$ & & 3 & 3 & 10 & 15 & 18 & 20 \\
$n$ & 1 & 6 & 4 & 14 & 9 & 17 & 19 \\
$40 \mathrm{~Hz}$ & & & & & & & \\
$\mu$ & 6 & 8 & 12 & 17 & 22 & 26 & 45 \\
$\sigma$ & 2 & 5 & 8 & 12 & 13 & 20 & 22 \\
$n$ & 4 & 9 & 7 & 12 & 9 & 18 & 19 \\
$80 \mathrm{~Hz}$ & & & & & & & \\
$\mu$ & 4 & 9 & 11 & 16 & 21 & 26 & 44 \\
$\sigma$ & 2 & 5 & 6 & 11 & 12 & 19 & 21 \\
$n$ & 2 & 6 & 7 & 11 & 9 & 17 & 18 \\
$160 \mathrm{~Hz}$ & & & & & & & \\
$\mu$ & 6 & 13 & 11 & 16 & 19 & 24 & 44 \\
$\sigma$ & & & 2 & 6 & 9 & 14 & 22 \\
$n$ & 1 & 1 & 4 & 7 & 8 & 14 & 12 \\
\hline
\end{tabular}

Columns in the table correspond to the spatial period of the grating and rows to the peak temporal frequency of the grating ridges. For each combination, $n$ indicates the number of afferents sampled, $\mu$ shows the average value of the $n$ mean cyclic discharge rates, and $\sigma$ the standard deviation.

The spatial period of the surface could be 1 of 7 possible values, namely, $0.75,1,1.25,1.5,1.75,2$, or $3 \mathrm{~mm}$. Twenty peak speeds were used, ranging from 15 to $480 \mathrm{~mm} / \mathrm{sec}$. For each surface the peak speeds were chosen so that the resulting peak temporal frequencies of the ridges were either 10,20,40, 80,160 , or $320 \mathrm{~Hz}$. Table 1 shows the corresponding values of the 3 parameters for all conditions used. As pointed out in Materials and Methods, it was impossible to use all conditions on all afferents.

\section{Responses at constant spatial period}

How do the responses of an SA change when the spatial period of the grating is kept constant and the peak speed is changed, and thus the peak temporal frequency of the grating ridges is also changed? This is illustrated by the cycle histograms in Figure 4 , which shows a matrix of responses for a typical SA. For the 1.5 and the $2 \mathrm{~mm}$ grating an increase in peak speed, and thus an increase in peak temporal frequency of the ridges, had a negligible effect on either the peak response or the mean response $(r)$ of the afferent. For the $3 \mathrm{~mm}$ grating, doubling the peak speed from 60 to $120 \mathrm{~mm} / \mathrm{sec}$ (which doubled the peak temporal frequency of the ridges from 20 to $40 \mathrm{~Hz}$ ) increased the mean response only from 30 to 33 impulses/sec. A 4-fold increase in peak speed from 60 to $240 \mathrm{~mm} / \mathrm{sec}$ (increase in peak temporal frequency from 20 to $80 \mathrm{IIz}$ ) increased the response from 30 to 44 impulses/sec. This trend was seen in all the SA afferents we studied: that is, if the spatial period of the surface remained constant, then the response of the SA remained essentially constant regardless of 8 -fold changes in the peak speed of the grating or corresponding 8-fold changes in the peak temporal frequency of the grating ridges. 
A.
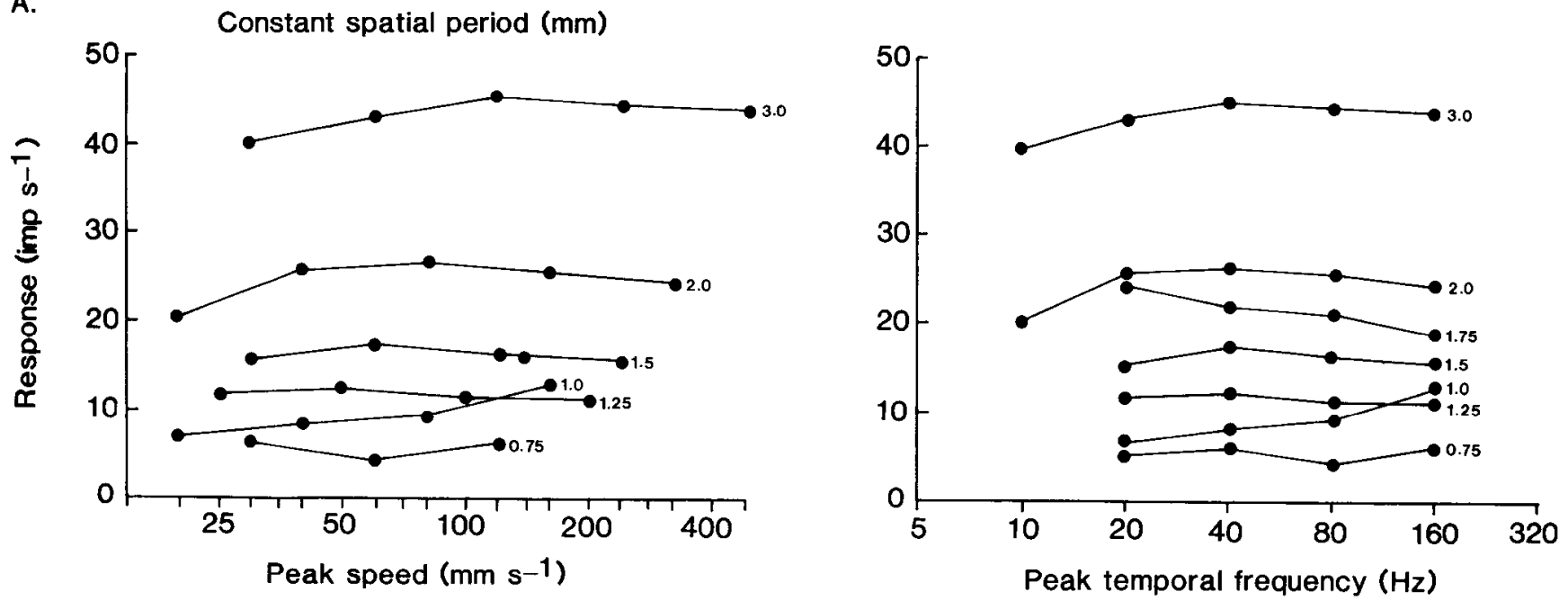

B.
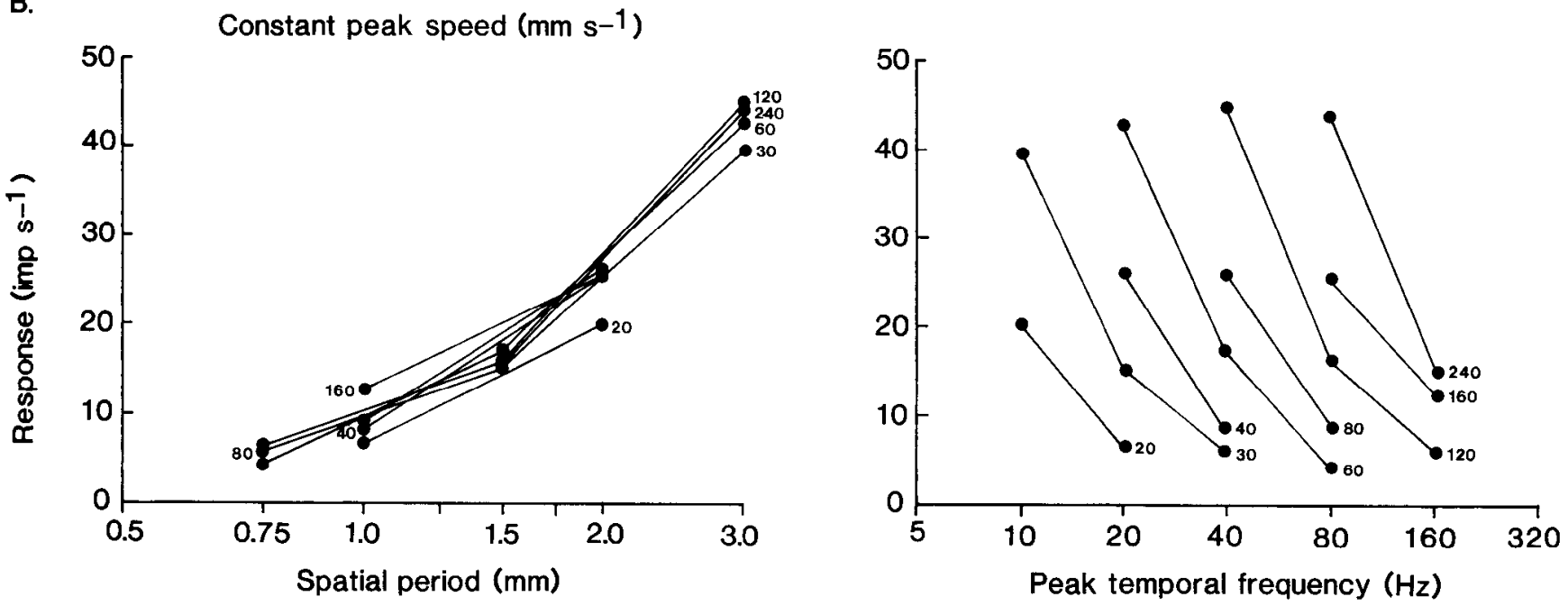

C.
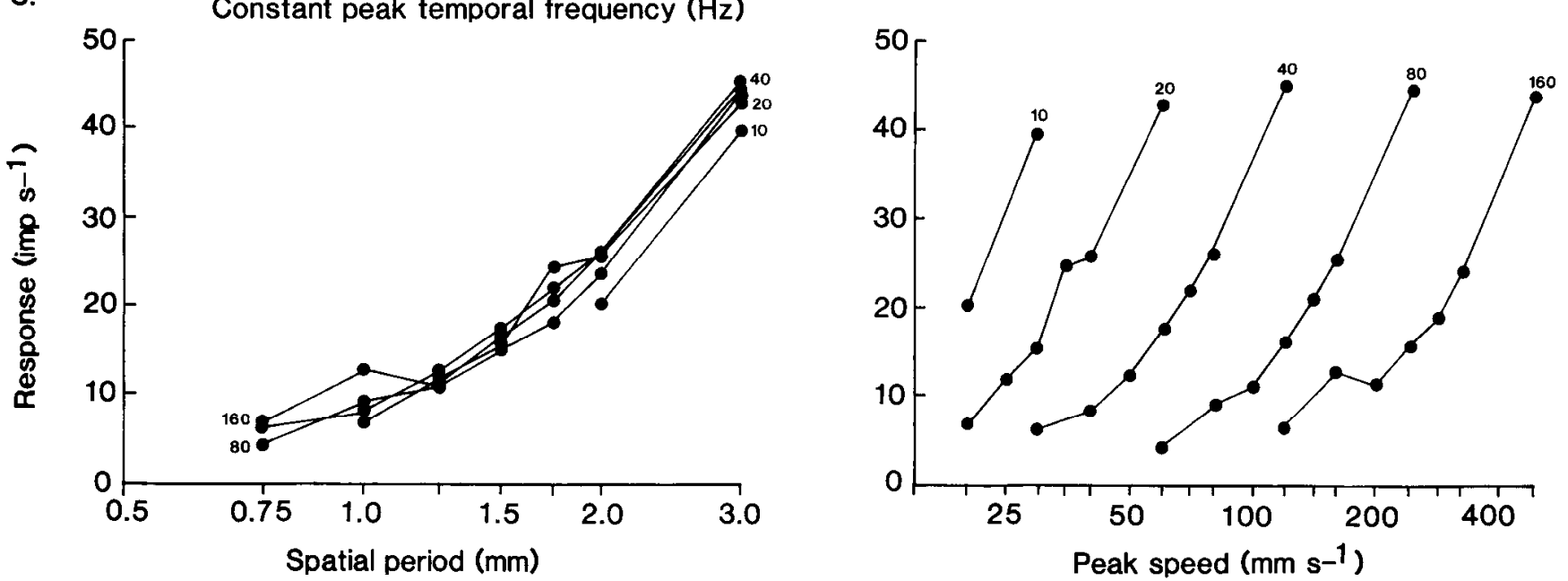

Figure 5. Average mean cyclic responses of the SA population. There are 3 stimulus variables-namely, grating spatial period, peak speed of movement, and the peak temporal frequency of the grating ridges-but only 2 of them (any 2) are independent. In the 3 sets of curves $A$, $B$, and $C$, one of the variables has been held constant and the response plotted as a function of the other 2 variables. $A$, Spatial period of the grating held constant at the value (in $\mathrm{mm}$ ) specified as the parameter for each line. $B$, Peak speed of movement held constant at the value (in mm/sec) specified as the parameter for each line. $C$, Peak temporal frequency of the grating ridges held constant at the value (in Hz) specified as the parameter for each line. The abscissae are logarithmic: The tick marks on the speed axis correspond to the values in Table 1 . The number of afferents contributing to each point and the SDs are given in Table 2. All curves can be plotted from Tables 2 and 1 . 


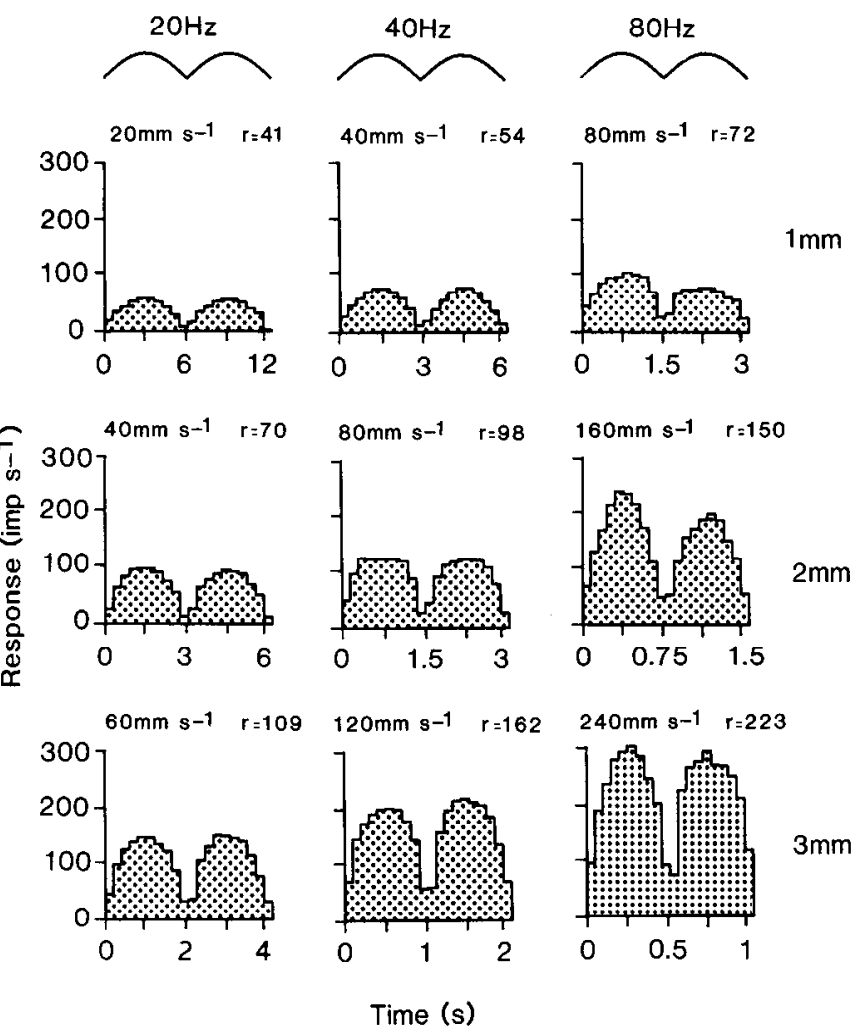

Figure 6. Matrix of cycle histograms for a typical RA. Format as in Figure 4. Moving from left to right in the 1 st, 2 nd, and 3 rd rows successively, the number of cycles averaged and bin widths for the 9 histograms are, respectively, 9 and $0.628 \mathrm{sec}, 12$ and $0.314 \mathrm{sec}, 9$ and $0.157 \mathrm{sec}, 11$ and $0.314 \mathrm{sec}, 10$ and $0.157 \mathrm{sec}, 13$ and $0.079 \mathrm{sec}, 9$ and $0.209 \mathrm{sec}, 10$ and $0.105 \mathrm{sec}$, and 18 and $0.052 \mathrm{sec}$.

The average relationship over the population of SAs is shown in Table 2. For each combination of parameters, Table 2 shows the number of afferents studied $(n)$, the average of their mean cyclic responses $(\mu)$, and the SD of their mean cyclic responses $(\sigma)$. For each afferent the mean cyclic response was calculated (see previous section), and the average and SD of this measure was calculated over the population. The major component of the $\mathrm{SD}$ was due to variation in sensitivity of individual SAs (see Discussion), and only a small component was due to experimental error or variation in the relationship between response and the stimulus parameters. Thus, it is more appropriate to show SDs than SEs here, emphasizing the distribution of responses in the natural SA population. Table 2 is arranged in columns at constant grating spatial period and rows at constant ridge peak temporal frequency: Corresponding peak speeds can be found in Table 1. The number of afferents sampled was not the same at all combinations and was greater for coarser gratings and lower peak speeds (lower peak temporal frequencies).

The response of the population can be seen most easily in Figure $5 A$. The peak speeds ranged from 15 to $480 \mathrm{~mm} / \mathrm{sec}$ and the peak temporal frequencies from 10 to $160 \mathrm{~Hz}$. Despite this large range of peak speeds and peak temporal frequencies, in general, they did not produce a change in the SA population response as long as the spatial period of the surface remained constant. No judgments can be made about the points at 160 $\mathrm{Hz}$ for the 0.75 and $1 \mathrm{~mm}$ gratings (peak speeds of 120 and 160 $\mathrm{mm} / \mathrm{sec}$, respectively) or the point at $20 \mathrm{~Hz}$ for the $0.75 \mathrm{~mm}$ grating (peak speed, $15 \mathrm{~mm} / \mathrm{sec}$ ) as only 1 afferent contributed

\begin{tabular}{|c|c|c|c|c|c|c|c|}
\hline \multirow{2}{*}{$\begin{array}{l}\text { Fre- } \\
\text { quency }\end{array}$} & \multicolumn{7}{|c|}{ Spatial period $(\mathrm{mm})$} \\
\hline & 0.75 & 1.0 & 1.25 & 1.5 & 1.75 & 2.0 & 3.0 \\
\hline \multicolumn{8}{|l|}{$10 \mathrm{~Hz}$} \\
\hline$\mu$ & & & & & & 53 & 64 \\
\hline$\sigma$ & & & & & & 3 & 15 \\
\hline$n$ & & & & & & 2 & 10 \\
\hline \multicolumn{8}{|l|}{$20 \mathrm{~Hz}$} \\
\hline$\mu$ & 32 & 38 & 44 & 50 & 62 & 69 & 86 \\
\hline$\sigma$ & & 3 & 9 & 24 & 22 & 23 & 26 \\
\hline$n$ & 1 & 2 & 2 & 11 & 9 & 16 & 16 \\
\hline \multicolumn{8}{|l|}{$40 \mathrm{~Hz}$} \\
\hline$\mu$ & 37 & 45 & 64 & 70 & 91 & 91 & 115 \\
\hline$\sigma$ & 19 & 25 & 28 & 33 & 31 & 33 & 33 \\
\hline$n$ & 16 & 27 & 9 & 24 & 9 & 22 & 18 \\
\hline \multicolumn{8}{|l|}{$80 \mathrm{~Hz}$} \\
\hline$\mu$ & 45 & 62 & 83 & 86 & 105 & 114 & 138 \\
\hline$\sigma$ & 37 & 36 & 43 & 44 & 42 & 45 & 41 \\
\hline$n$ & 20 & 17 & 9 & 18 & 8 & 17 & 17 \\
\hline \multicolumn{8}{|l|}{$160 \mathrm{~Hz}$} \\
\hline$\mu$ & 51 & 72 & 100 & 97 & 126 & 128 & 155 \\
\hline$\sigma$ & 47 & 50 & 57 & 60 & 63 & 57 & 44 \\
\hline$n$ & 19 & 16 & 9 & 18 & 9 & 17 & 16 \\
\hline \multicolumn{8}{|l|}{$320 \mathrm{~Hz}$} \\
\hline$\mu$ & 53 & 79 & 113 & 116 & & & \\
\hline$\sigma$ & 45 & 50 & 63 & 65 & & & \\
\hline$n$ & 13 & 12 & 6 & 12 & & & \\
\hline
\end{tabular}

Format as in Table 2.

to each of these points. In fact, since the sensitivity of afferents varied greatly and since the pool of afferents was different at different points on the curve, great care must be taken in making specific comparisons (see Discussion), and only the general trends should be studied in these curves.

Figure 6 shows a matrix of cycle histograms for a typical RA, analogous to Figure 4 for the SA. For all 3 gratings, an increase in peak speed (and thus an increase in ridge peak temporal frequency) was accompanied by an increase in both the peak response and the mean cyclic response. This is in contrast to the behavior of the typical SA illustrated in Figure 4. To assess the characteristics of the RA population for each combination of stimulus parameters, the average value of the mean cyclic discharge rate was calculated. These values are shown in Table 3 , together with the SDs of the values and the number of afferents studied at each combination. Table 3 for the RAs is equivalent to Table 2 for the SAs. Again, the number of afferents was different for different stimulus combinations, being least at low peak temporal frequencies and small spatial periods. The RA population responses to gratings of constant spatial period are most easily seen in Figure $7 \mathrm{~A}$, where they are shown as a function of peak speed on the left and as a corresponding function of peak temporal frequency of the grating ridges on the right. The clear trend of an increase in response with an increase in peak speed of the grating (and concomitant increase in peak temporal frequency of the ridges) is in marked contrast to the unchanging responses of the SA population (Fig. $5 A$ ).

A matrix of cycle histograms for a typical $\mathrm{PC}$ is shown in Figure 8 . For the 1 and $2 \mathrm{~mm}$ gratings, both the peak response 

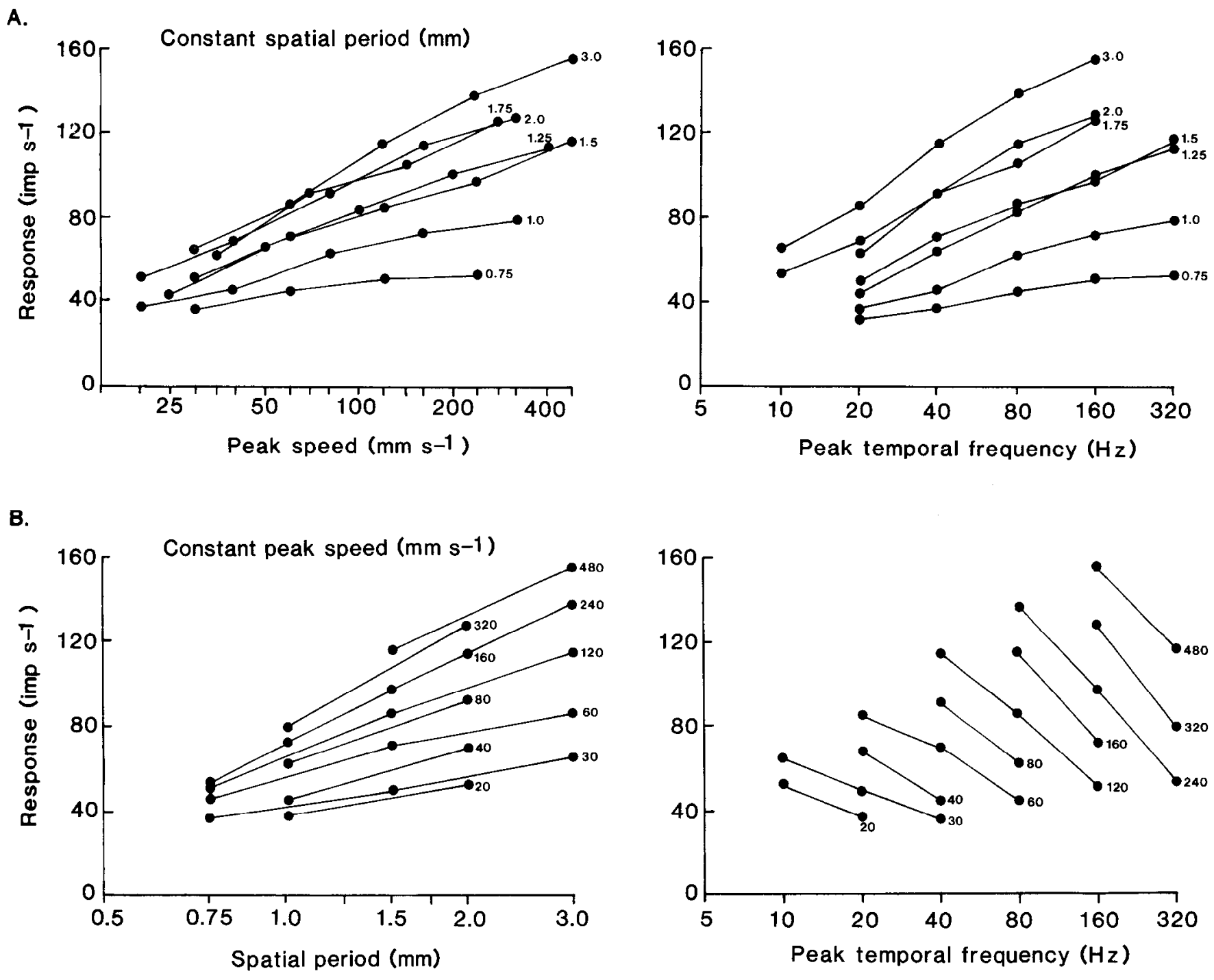

c.
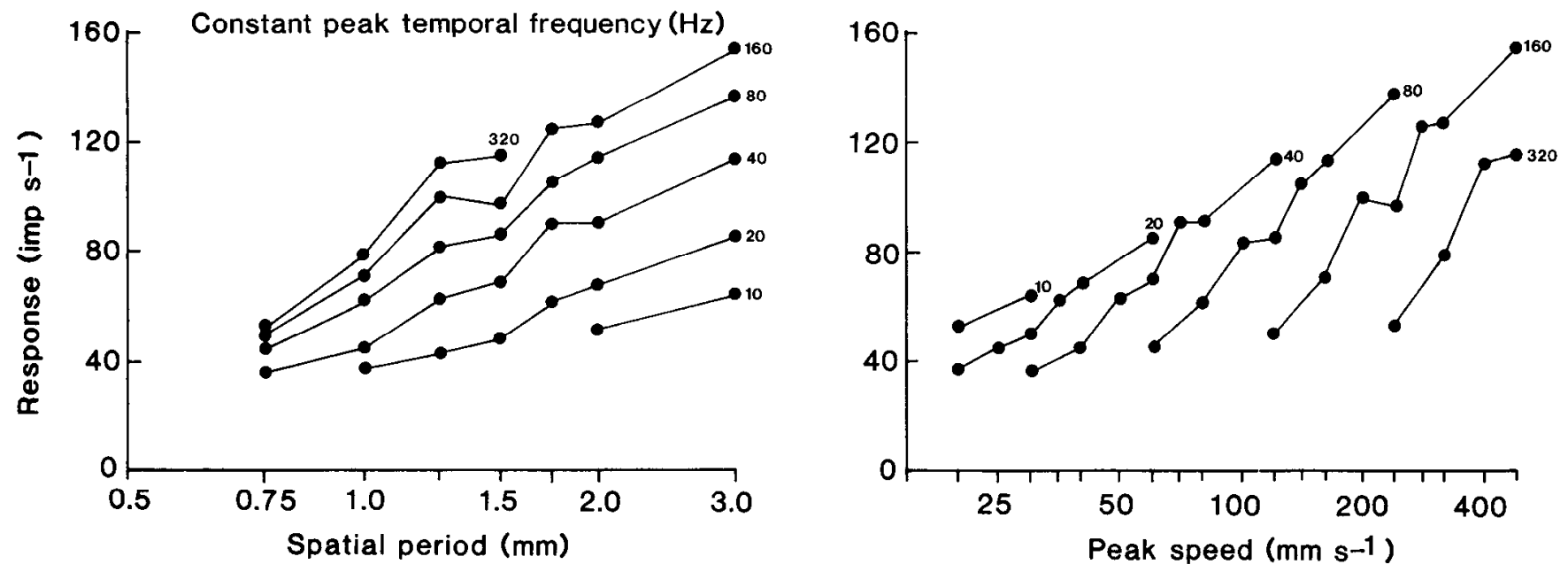

Figure 7. Average mean cyclic responses of the RA population. Format as in Figure 5. The number of afferents contributing to each point and the SDs are given in Table 3. 


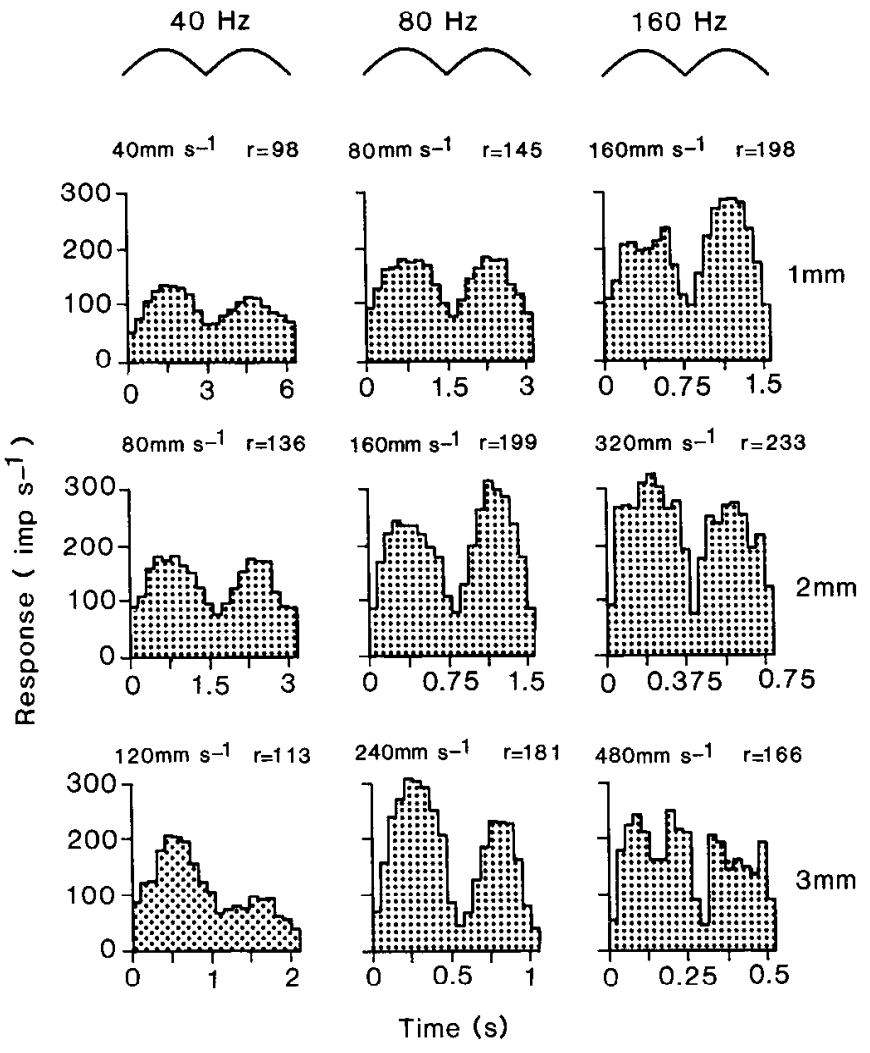

Figure 8. Matrix of cycle histograms for a typical PC. Format as in Figure 4. Moving from left to right in the 1 st, 2 nd, and 3 rd rows successively, the number of cycles averaged and bin widths for the 9 histograms are, respectively, 7 and $0.314 \mathrm{sec}, 10$ and $0.157 \mathrm{sec}, 20$ and $0.079 \mathrm{sec}, 13$ and $0.157 \mathrm{sec}, 20$ and $0.079 \mathrm{sec}, 20$ and $0.039 \mathrm{sec}, 21$ and $0.105 \mathrm{sec}, 22$ and $0.052 \mathrm{sec}$, and 33 and $0.026 \mathrm{sec}$.

and the mean cyclic response increased with an increase in the peak speed and thus with the accompanying increase in the peak temporal frequency of the grating ridges. For the $3 \mathrm{~mm}$ grating, an increase in peak speed from 120 to $240 \mathrm{~mm} / \mathrm{sec}$ (40 to 80 $\mathrm{Hz}$ ) produced an increase in the peak and mean cyclic responses, but further increase to $480 \mathrm{~mm} / \mathrm{sec}(160 \mathrm{~Hz})$ reduced the responses slightly. Although this $\mathrm{PC}$ is, in general, typical of our sample of PCs, there was much greater variability in the responses of individual PCs than in the responses of individual SAs or RAs. Table 4 shows the average responses over our population of PCs together with SDs and the numbers of afferents at each combination of stimulus parameters. These population responses must be interpreted with some care not only because of the larger variation within the $\mathrm{PC}$ responses, but also because of the small number of afferents in our sample. Nevertheless, it is clear from Figure $9 A$ that, for a constant grating spatial period, the response in the $\mathrm{PC}$ population increased as the peak speed of the grating increased and hence as the concomitant peak temporal frequency of the grating ridges increased. In this regard the $\mathrm{PC}$ population resembles the RA population.

\section{Responses at constant peak speed}

For the SA illustrated in Figure 4, comparison of the center histogram in the top row with the left histogram in the bottom rows shows that when the peak speed remained constant at 60 $\mathrm{mm} / \mathrm{sec}$, increasing the spatial period of the grating from 1.5 to
$3 \mathrm{~mm}$ increased both the peak and mean responses. This increase in spatial period, with speed held constant, caused a concomitant decrease in the peak temporal frequency of the ridges from 40 to $20 \mathrm{~Hz}$. The same effect can be seen by comparing the right histogram in the top row with the center histogram in the bottom row. Here, at $120 \mathrm{~mm} / \mathrm{sec}$, an increase in response resulted when the spatial period increased from 1.5 to $3 \mathrm{~mm}$, with a corresponding decrease in peak temporal frequency of the ridges from 80 to $40 \mathrm{~Hz}$. From Tables 2 and 1 the average responses of the population of SAs can be calculated at each peak speed, and these are plotted in Figure $5 B$. The left of Figure $5 B$ is, in fact, a replotting of the left of Figure $5 A$ with the constant parameter and the variable interchanged. The right of Figure $5 B$ is derived from the right of Figure $5 A$ via Table 1. As can be seen in Tables 2-4, the matrix of variables used in these experiments covered a number of grating spatial periods and, at each spatial period covered, the same peak temporal frequencies of the ridges of $10,20,40,80,160$, and $320 \mathrm{~Hz}$. Thus, the same speeds were not used with all gratings: for example, at $30 \mathrm{~mm} / \mathrm{sec}$, a $3 \mathrm{~mm}$ grating had a peak temporal frequency of $10 \mathrm{~Hz}$ (see Table 1), and this combination was used; in contrast, at $30 \mathrm{~mm} / \mathrm{sec}$ a $2 \mathrm{~mm}$ grating had a peak temporal frequency of $15 \mathrm{~Hz}$, and thus this combination was not used.'

The behavior of the SA population is clearly seen in Figure $5 B$. When the peak speed of movement remained constant, an increase in the spatial period of the grating (left figure) produced an increase in the average response of the population. Alternatively, as portrayed in the right figure, an increase in the peak temporal frequency of the ridges caused a decrease in the SAs' responses when peak speed was not altered.

The behavior of a typical RA when the peak speed of movement remained constant can be seen in the matrix of responses in Figure 6. Comparing the center histogram of the top row with the left histogram of the middle row shows that with peak speed contant at $40 \mathrm{~mm} / \mathrm{sec}$, an increase in the spatial period of the grating from 1 to $2 \mathrm{~mm}$ caused an increase in the peak and mean cyclic responses. The increase in spatial period was accompanied by a decrease in the peak temporal frequency of the ridges from 40 to $20 \mathrm{~Hz}$. Similarly, at $80 \mathrm{~mm} / \mathrm{sec}$ (right histogram in top row and center histogram in middle row), the response increased when the spatial period increased from 1 to 2 $\mathrm{mm}$ and thus when the peak temporal frequency of the ridges decreased from 80 to $40 \mathrm{~Hz}$. Figure $7 \mathrm{~B}$ shows the average responses of the RA population derived from Tables 3 and 1 . When the peak speed of movement remained constant, the responses of the RA afferents increased with an increase in the spatial period of the grating (left figure) and thus decreased with a increase in the peak temporal frequency of the grating ridges (right figure). In this respect, the SA population and the RA population behave similarly.

The responses of the PCs when the peak speed of movement remained constant was less predictable than the responses of the SAs or RAs. This can be seen in Figure 8, where at $80 \mathrm{~mm}$ / sec (center histogram in top row and left histogram in middle row) an increase in spatial period from 1 to $2 \mathrm{~mm}$ (decrease in

\footnotetext{
'For similar reasons some points are included in Figure $5 A$ but not in Figure $5 B$. For example, the point with the combination of parameters spatial period $=$ $1.25 \mathrm{~mm}$, peak speed $=100 \mathrm{~mm} / \mathrm{sec}$, and peak temporal frequency $=80 \mathrm{~Hz}$ is included in Figure $5 \mathrm{~A}$ as there are other points on the line spatial period $=1.25$ $\mathrm{mm}$ but is not included in Figure $5 B$ as there are no other points at a speed of $100 \mathrm{~mm} / \mathrm{sec}$
} 
A.

Constant spatial period (mm)

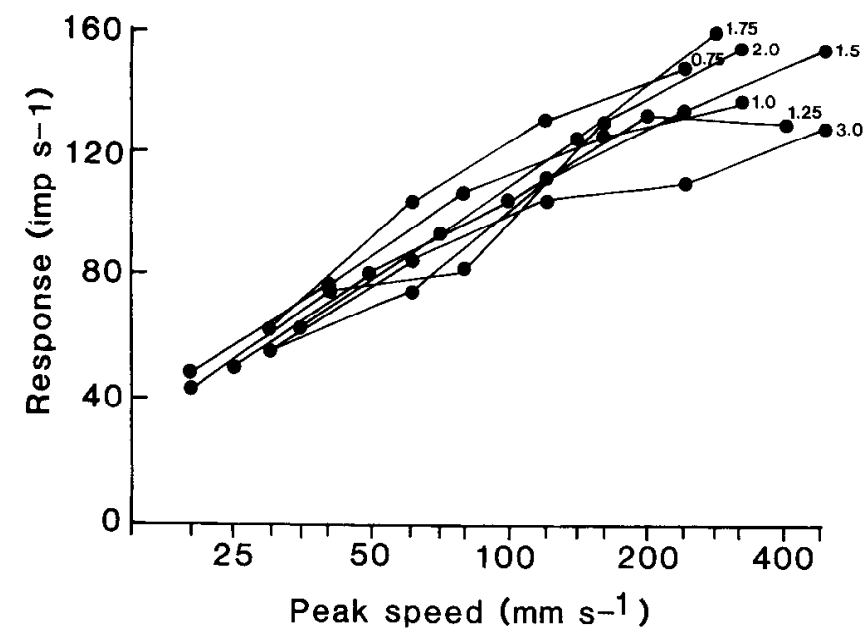

B.

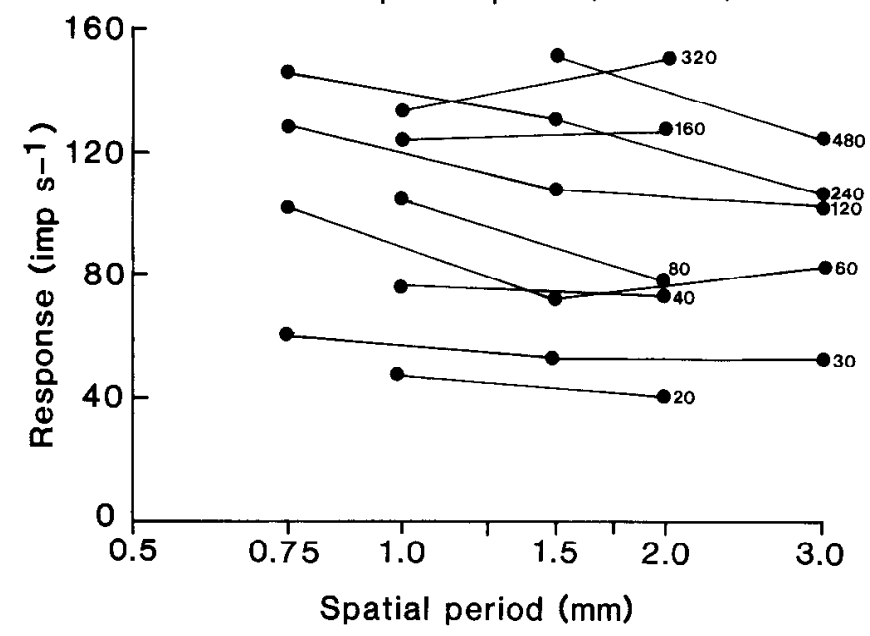

C.

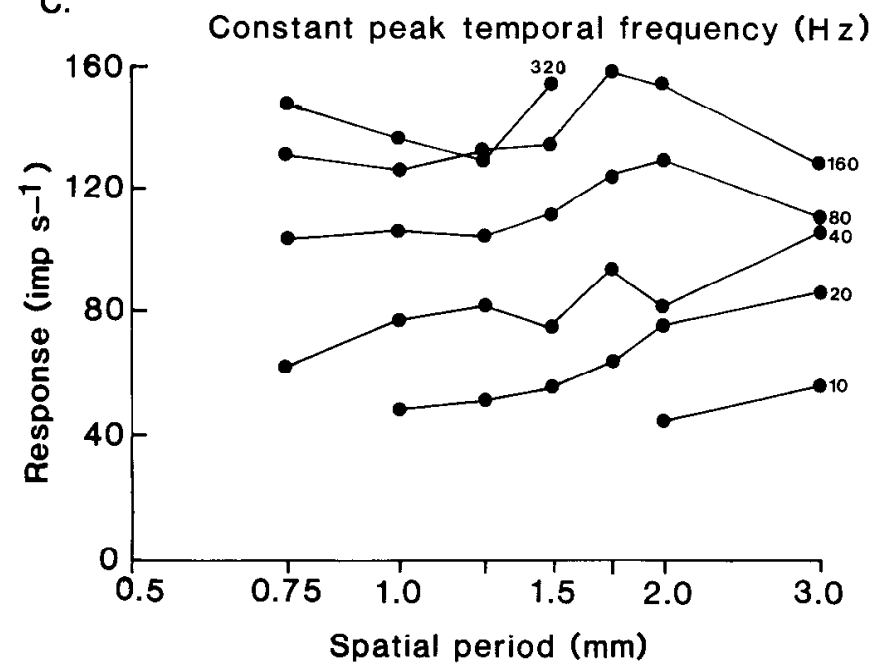

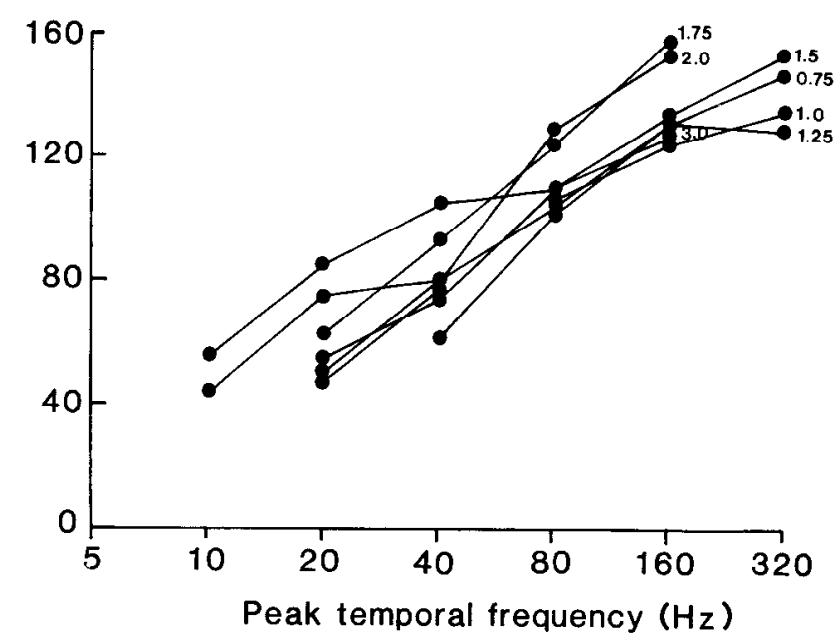
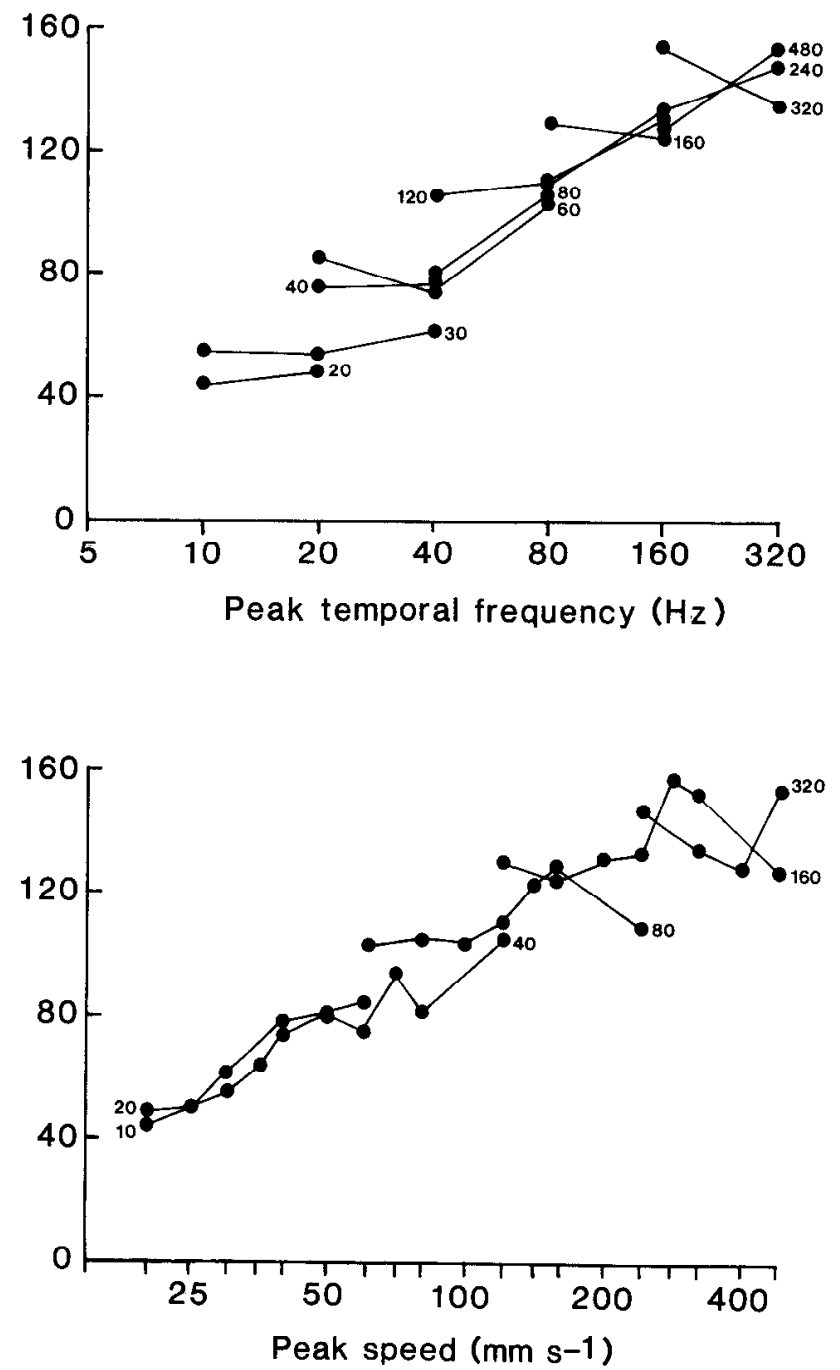

Figure 9. Average mean cyclic responses of the PC population. Format as in Figure 5. The number of afferents contributing to each point and the SDs are given in Table 4. 
Table 4. Average response (impulses/sec) of the $\mathrm{PC}$ population for the various combinations of stimulus parameters

\begin{tabular}{|c|c|c|c|c|c|c|c|}
\hline \multirow{2}{*}{$\begin{array}{l}\text { Fre- } \\
\text { quency }\end{array}$} & \multicolumn{7}{|c|}{ Spatial period (mm) } \\
\hline & 0.75 & 1.0 & 1.25 & 1.5 & 1.75 & 2.0 & 3.0 \\
\hline \multicolumn{8}{|l|}{$10 \mathrm{~Hz}$} \\
\hline$\mu$ & & & & & & 44 & 55 \\
\hline$\sigma$ & & & & & & & 28 \\
\hline$n$ & & & & & & 1 & 3 \\
\hline \multicolumn{8}{|l|}{$20 \mathrm{H} L$} \\
\hline$\mu$ & & 49 & 50 & 55 & 63 & 76 & 85 \\
\hline$\sigma$ & & & & 38 & 53 & 34 & 16 \\
\hline$n$ & & 1 & 1 & 3 & 2 & 6 & 6 \\
\hline \multicolumn{8}{|l|}{$40 \mathrm{~Hz}$} \\
\hline$\mu$ & 62 & 77 & 81 & 75 & 94 & 81 & 105 \\
\hline$\sigma$ & 54 & 47 & 64 & 37 & 60 & 34 & 22 \\
\hline$n$ & 4 & 7 & 3 & 7 & 2 & 6 & 7 \\
\hline \multicolumn{8}{|l|}{$80 \mathrm{~Hz}$} \\
\hline$\mu$ & 104 & 106 & 104 & 110 & 124 & 129 & 110 \\
\hline$\sigma$ & 49 & 61 & 90 & 49 & 67 & 57 & 35 \\
\hline$n$ & 7 & 7 & 3 & 7 & 2 & 6 & 7 \\
\hline \multicolumn{8}{|l|}{$160 \mathrm{~Hz}$} \\
\hline$\mu$ & 131 & 125 & 132 & 134 & 158 & 154 & 128 \\
\hline$\sigma$ & 66 & 79 & 118 & 69 & 56 & 52 & 21 \\
\hline$n$ & 7 & 7 & 3 & 7 & 2 & 7 & 7 \\
\hline \multicolumn{8}{|l|}{$320 \mathrm{~Hz}$} \\
\hline$\mu$ & 147 & 136 & 129 & 153 & & & \\
\hline$\sigma$ & 111 & 99 & 107 & 68 & & & \\
\hline$n$ & 7 & 7 & 3 & 7 & & & \\
\hline
\end{tabular}

Format as in Table 2 .

peak temporal frequency of the ridges from 80 to $40 \mathrm{~Hz}$ ) caused a slight decrease in response. On the other hand, at $160 \mathrm{~mm} /$ sec (right histogram in top row and center histogram in middle row) an increase in spatial period from 1 to $2 \mathrm{~mm}$ (decrease in peak temporal frequency of the ridges from 160 to $80 \mathrm{~Hz}$ ) produced a negligible change in response. The average response of the PC population, derived from Tables 4 and 1 , is shown in Figure $9 B$. There was a trend towards a decrease in response with an increase in the grating spatial period or, equivalently, an increase in response with an increase in the peak temporal frequency of the ridges. This. result is the opposite of that seen in the SA and RA populations. However, this effect was slight and variable; for example, the opposite trend was seen at 320 and $160 \mathrm{~mm} / \mathrm{sec}$. Particularly in view of the small sample of PCs, we do not regard our PC population response as varying consistently with the spatial period of the grating or with the peak temporal frequency of the ridges when the peak speed was held constant.

\section{Responses at constant peak temporal frequency of the grating ridges}

In Figure 4 the peak temporal frequency of the grating ridges remains constant within cach column of histograms for the typical SA illustrated. Proceeding down each column (constant peak temporal frequency), the peak and mean responses increase as the spatial period of the grating increases or, equivalently, as the peak speed of movement increases. The average response of the SA population at constant peak temporal frequency of the ridges is plotted in Figure $5 C$. There was a marked increase in the SA population responsc with an increase in the grating spatial period or with an increase in the peak speed of movement.

Similarly, for the typical RA illustrated in Figure 6, within columns of constant peak temporal frequency of the ridges, the peak and mean responses increase with an increase in the spatial period of the grating or, equivalently, as the peak speed of movement increases. This relationship is shown graphically for the average response of the RA population in Figure $7 \mathrm{C}$. In this regard, the RA population behaved similarly to the SA population.

For the typical PC illustrated in Figure 8, the changes in response were more complex. As an example, at a peak temporal frequency of the ridges of $80 \mathrm{~Hz}$ (center column), a change in grating spatial period from 2 to $3 \mathrm{~mm}$ caused an increase in peak response for the first half cycle and a decrease in peak response for the second half cycle (opposite direction of movement). The mean response over the whole cycle decreased. The change of response pattern within a cycle is dealt with in a companion paper (Morley and Goodwin, 1987), and here we consider only the mean cyclic response. For this $\mathrm{PC}$, at all 3 peak temporal frequencies of the ridges $(40,80$, and $160 \mathrm{~Hz})$, the mean cyclic response to a $2 \mathrm{~mm}$ grating was larger than the response to either a 1 or a $3 \mathrm{~mm}$ grating. The average responses of the PC population are illustrated in Figure $9 C$. At peak temporal frequencies of 20 and $40 \mathrm{~Hz}$, the trend in the PC population was towards an increase in response with an increase in the spatial period of the grating or, equivalently, with an increase in the peak speed of movement. At 80 and $160 \mathrm{~Hz}$ the response increased up to periods of about $2 \mathrm{~mm}$ (up to peak speeds of about 160 and $320 \mathrm{~mm} / \mathrm{sec}$, respectively) and then decreased with an increase in spatial period or peak speed. Again, because of the small sample of afferents at many of the points, only the major trends of these data should be interpreted.

\section{Discussion}

Changes in responses of the afferent populations with changes in stimulus variables

In these experiments the characteristics of the textured surface were varied systematically by varying the spatial period of the grating. The characteristics of the movement between the surface and the skin were varied systematically by varying the peak speed of the sinusoidal motion. Either of these changes produced a concomitant change in the peak temporal frequency at which grating ridges passed over the fingertip. (To be precise we should refer to the temporal frequency of the grating spatial cycle: We do not impute responses to ridges rather than grooves but simply use ridge frequency as a convenient description.) The manner in which the responses of the mechanoreceptive afferent populations changed with the above changes in the stimulus is depicted in Figures 5, 7, and 9 for the SA, RA, and PC populations, respectively. In these curves the response measure used is the mean response per stimulus (sinusoidal) cycle averaged over the whole population of afferents studied. Alternative response measures are discussed in an accompanying paper (Morley and Goodwin, 1987).

Any 2 of the 3 variables, namely, the spatial period of the grating, the peak speed of the sinusoidal movement, and the peak temporal frequency of the grating ridges may be considered as independent, and the remaining dependent variable is defined by the following relationship: peak temporal frequency $=$ peak speed/spatial period. Thus, the responses of the afferent popu- 
lations are best described by a full set of parametric curves (Figs. 5,7 , and 9) showing how the responses vary when one of the parameters is held constant and the other 2 covary in accordance with the above relationship.

Figure $5 \mathrm{~A}$ shows that when the spatial period of the grating was held constant, the response of the SA population was essentially invariant with changes in the peak speed of movement or, equivalently, in the peak temporal frequency of the ridges. In contrast, Figures $7 A$ and $9 A$ show that the responses of both the RA and PC populations increased with an increase in peak speed or an increase in peak temporal frequency of the ridges. Thus, in this respect the RA and PC populations behaved similarly and the SA population differently. Figures $5 B$ and $7 B$ show that when the peak speed of movement was held constant, the responses of both the SA and RA populations increased with an increase in the spatial period of the grating or decreased with an increase in the peak temporal frequency of the ridges. In contrast, for the PC population, the response was minimally affected, if anything tending to decrease with an increase in spatial period or increase with an increase in peak temporal frequency of the ridges. Thus in this respect the SA and RA populations behaved similarly and the PC population differently. However, the slope of the curves is different for the SA and RA populations. Finally, Figures $5 C, 7 C$, and $9 C$ show that, for all 3 populations, when the peak temporal frequency of the ridges was held constant the response increased with an increase in the spatial period of the grating or increased with an increase in the peak speed of movement. In this respect all 3 populations behaved similarly, although, again, the slopes of the curves are not the same for different populations.

These charactcristics of the 3 cutaneous, afferent populations could not be predicted from previous data. Lamb (1983b, fig. 3) observed that when a pattern of large dots was moved over the fingertip, an increase in the speed of movement increased the mean response of the RA and PC populations but did not affect the response of the SA population. This is consistent with Figures $5 A, 7 A$, and $9 A$. For punctate stimuli it is well established, both from direct measurements (Pubols and Pubols, 1976) and from vibratory tuning curves (Freeman and Johnson, 1982), that RAs and PCs are more dependent on probe velocity than SAs. It is tempting to conclude that these observations are consistent with our data, but it is difficult to compare responses to vibrating probes with those to moving gratings: Without a detailed spatiotemporal model of the skin mechanics and receptor properties, we feel that such comparisons are not warranted.

\section{Independent representation of stimulus variables in the population responses}

Are there any aspects of the population responses that could provide the CNS with information about the spatial period of the grating, regardless of the peak speed of movement? It is clear from Figure 5 that the SA population provided such information as its response (within experimental error) depended only on the spatial period of the grating and not on the peak speed of movement or on the peak temporal frequency of the ridges. The RA and PC population responses depended on all 3 parameters of the stimulus. However, if the ratios of RA and PC responses are plotted (ratio of Figs. $7 A$ and $9 A$ ), then these ratios are relatively invariant with the peak speed of movement and with the peak temporal frequency of the ridges. Thus, the ratio of the RA and PC population responses also provided the CNS with information about the spatial period of the grating, re- gardless of the peak speed of movement or the peak temporal frequency of the ridges. In summary, under the experimental conditions used here, both the SA population response and the ratio of the RA and $\mathrm{PC}$ population responses provide unambiguous information about the grating spatial period. Thus, although trivially in this case, the response ratio SA:RA:PC also provides such information.

None of the responses shown in Figures $5 B, 7 B$, and $9 B$ unequivocally reflect changes in the peak speed of movement, regardless of the spatial period of the grating or of the peak temporal frequency of the ridges. The PC population (Fig. 9B) approaches this condition, but the trends are too variable to provide this information without the assistance of an additional mechanism. Moreover, if the ratios of the RA and SA responses (ratio of Figs. $7 B$ and $5 B$ ) are plotted, then these ratios also do not provide such information. However, since the brain can establish the spatial period of the grating by the means discussed above, it can easily extract information about the peak speed of movement from the RA population (in effect from Fig. $7 \mathrm{~A}$, left). In addition, sequential activation of afferents with adjacent receptive fields provides, at least in principle, a measure of the peak speed of movement and indeed of the complete movement profile (Darian-Smith and Oke, 1980). However, for sinusoidal movement, such a cue depends in a complex way on factors such as innervation density and the similarity of discharge profiles in adjacent afferents and may not really be useful.

None of the responses in Figures $5 C, 7 C$, and $9 C$ reflect changes in the peak temporal frequency of the ridges regardless of the spatial period of the grating or the peak speed of movement, and no simple ratios of the 3 population responses will provide such information. Ilere, too, the $\mathrm{PC}$ population (Fig. $9 \mathrm{C}$ ) approaches this condition, but the variability of the trends necessitates an additional mechanism as well. Once again, since the brain can establish the spatial period of the grating, it can easily establish the peak temporal frequency of the ridges from the RA population response (in effect from Fig. $7 A$, right). Also, the pattern of the afferent discharges within a cycle of movement contains a clear and independent measure of the peak temporal frequency of the ridges, as shown in the companion paper (Morley and Goodwin, 1987).

\section{Resolution in the population responses}

Figures 5, 7, and 9 show the mean responses in a stimulus (sinusoidal) cycle averaged over the population, and Tables 24 show the SDs of these values and the number of afferents studied at each stimulus condition. The SDs indicate the considerable variation in sensitivity or responsiveness among the afferents of each population and are consistent with the variation in sensitivity to vibratory stimuli reported by other investigators (Talbot et al., 1968). These SDs are not indicative of the resolution in the population responses. Consider, as an illustration, the possible contribution of the SA population in discriminating 2 gratings with spatial periods of 2 and $3 \mathrm{~mm}$, respectively, assuming that the peak temporal frequency of the ridges is held constant at $20 \mathrm{~Hz}$. Table 2 shows that the average responses of the SA population were 26 and $43 \mathrm{impulses} / \mathrm{sec}$, respectively, and the SDs were 18 and $20 \mathrm{impulses} / \mathrm{sec}$, respectively. In practice, any discrimination paradigm would invariably involve moving each grating successively over the same area of skin, thus engaging the same afferents. The relative change in the response of each afferent would be similar, regardless of the sensitivity of that afferent, and the resolution would not be 
affected by the distribution of sensitivities per se. In the above example, if the ratio of the responses to the 3 and $2 \mathrm{~mm}$ grating are calculated for each afferent in our sample, the average value and SD of this ratio are 2.10 and 0.617 , respectively. On the basis of a $t$ test, the number of SA afferents needed to discriminate the $3 \mathrm{~mm}$ grating from the $2 \mathrm{~mm}$ grating is only $6(p<$ 0.01 ). When a human subject performs such a discrimination, the number of SAs engaged is on the order of 200 (Darian-Smith and Kenins, 1980; Morley et al., 1983). In these experiments we have concentrated on gathering data for a large number of gratings with relatively large increments in spatial period and have not attempted to assess the resolution in the population responses (Johnson, 1980) for the 5\% changes in spatial period that can be discriminated by the human.

\section{References}

Chubbuck, J. G. (1966) Small motion biological stimulator. APL Technical Digest 5: 18-23.

Darian-Smith, I., and P. Kenins (1980) Innervation density of mechanoreceptive fibres supplying glabrous skin of the monkey's index finger. J. Physiol. (Lond.) 309: 147-155.

Darian-Smith, I., and L. E. Oke (1980) Peripheral neural representation of the spatial frequency of a grating moving across the monkey's finger pad. J. Physiol. (Lond.) 309: 117-133.

Darian-Smith, I., I. Davidson, and K. O. Johnson (1980) Peripheral neural representation of spatial dimensions of a textured surface moving across the monkey's finger pad. J. Physiol. (Lond.) 309: 135-146.

Darian-Smith, I., A. Goodwin, M. Sugitani, and J. Heywood (1985) Scanning a textured surface with the fingers: Events in sensorimotor cortex. In Hand Function and the Neocortex, A. W. Goodwin and I. Darian-Smith, eds., pp. 17-43, Springer-Verlag, Heidelberg.

Day, R. H., and R. G. Dickenson (1979) Learning to identify Braille numerals with active and passive touch. Research Note, Monash University, Australia.

Freeman, A. W., and K. O. Johnson (1982) Cutaneous mechanoreceptors in macaque monkey: Temporal discharge patterns evoked by vibration, and a receptor model. J. Physiol. (Lond.) 323: 21-41.
Goodwin, A. W., B. D. Youl, and N. P. Zimmerman (1981) Single quickly adapting mechanoreceptive afferents innervating monkey glabrous skin: Response to two vibrating probes. J. Neurophysiol. 45 : 227-242.

Goodwin, A. W., J. W. Morley, C. Clarke, B. Lumaksana, and I. DarianSmith (1985) A stimulator for moving textured surfaces sinusoidally across the skin. J. Neurosci. Methods 14: 121-125.

Johansson, R. S. (1978) Tactile sensibility in the human hand: Receptive field characteristics of mechanoreceptive units in the glabrous skin area. J. Physiol. (Lond.) 281: 101-123.

Johnson, K. O. (1980) Sensory discrimination: Neural processes preceding discrimination decision. J. Neurophysiol. 43: 1793-1815.

Johnson, K. O., and G. D. Lamb (1981) Neural mechanisms of spatial tactile discrimination: Neural patterns evoked by braille-like dot patterns in the monkey. J. Physiol. (Lond.) 310: 117-144.

Lamb, G. D. (1983a) Tactile discrimination of textured surfaces: Psychophysical performance measurements in humans. J. Physiol. (Lond.) 338: 551-565.

Lamb, G. D. (1983b) Tactile discrimination of textured surfaces: Peripheral neural coding in the monkey. J. Physiol. (Lond.) 338: 567587.

Lederman, S. J. (1981) The perception of surface roughness by active and passive touch. Bull. Psychonomic Soc. 18: 253-255.

Lederman, S. J., and M. M. Taylor (1972) Fingertip force, surface geometry, and the perception of roughness by active touch. Percept. Psychophys. 12: 401-408.

Morley, J. W., and A. W. Goodwin (1987) Sinusoidal movement of a grating across the monkey's fingerpad: Temporal patterns of afferent fiber responses. J. Neurosci., 7: 2181-2191.

Morley, J. W., A. W. Goodwin, and I. Darian-Smith (1983) Tactile discrimination of gratings. Exp. Brain Res. 49: 291-299.

Phillips, J. R., and K. O. Johnson (1981) Tactile spatial resolution. II. Neural representation of bars, edges, and gratings in monkey primary afferents. J. Neurophysiol. 46: 1192-1203.

Pubols, B. H., Jr., and L. M. Pubols (1976) Coding of mechanical stimulus velocity and indentation depth by squirrel monkey and raccoon glabrous skin mechanoreceptors. J. Neurophysiol. 39: 773-787.

Talbot, W. H., I. Darian-Smith, H. H. Kornhuber, and V. B. Mountcastle (1968) The sense of flutter-vibration: Comparison of the human capacity with response patterns of mechanoreceptive afferents from the monkey hand. J. Neurophysiol. 31: 301-334. 